UH-511-1130-08

UCI-2008-31

\title{
A Toy Model for Gauge-Mediation in Intersecting Brane Models
}

\author{
Jason Kumar \\ Department of Physics and Astronomy, University of Hawaii \\ Honolulu, HI 96822, USA \\ and \\ Department of Physics, University of California, Irvine \\ Irvine, CA 92697, USA
}

\begin{abstract}
We discuss the phenomenology of a toy intersecting brane model where supersymmetry is dynamically broken in an open string hidden sector and gauge-mediated to the visible sector. Scalar masses $\sim \mathrm{TeV}$ are easily realizable, and R-symmetry is broken. These ideas are easily generalizable to other intersecting brane models.
\end{abstract}

August 2008 


\section{Introduction}

To utilize supersymmetry to understand the electroweak scale, there are two broad questions which one must answer. Although supersymmetry stabilizes the scale of electroweak symmetry breaking against quantum corrections, it does not itself explain why that scale is small in relation to an a priori more natural scale such as the GUT (or Planck) scale. Thus, there has been much study of models where dynamics generates supersymmetry-breaking at a scale exponentially smaller than the natural scale of the theory [1]. In addition, it has long been known that supersymmetry cannot be broken within the MSSM sector itself. Instead, it must be broken in some auxiliary sector, with its effects communicated to MSSM sector via interactions. There are several different models for this type of mediation of supersymmetrybreaking (for example, through gravitational interactions or through gauge interactions), but each such mechanism has its advantages and difficulties.

There have been recent attempts to tackle both of these problems [2, 3, 4]. String theory has a natural role to play in understanding these questions. In a low-energy effective field theory, the parameters of the theory are simply given at the energy-scale where the theory is defined; a real understanding of the scale of those parameters depends on the microscopic physics which has been integrated out. If one has specific models of a high-energy theory, then one can potentially find that certain parameters are necessarily small, leading to a small SUSY-breaking scales [5. String theoretic realizations of the Standard Model are a natural starting point.

These classes of microscopic theories connect the question of how SUSY is broken to the question of how SUSY-breaking is mediated to the MSSM sector. For example, the two most natural ways of embedding the MSSM within Type II string theory are by branes at singularities or by intersecting branes. In the former class gravity mediation occurs rather generically, whereas in the latter class, gauge-mediated supersymmetry-breaking (GMSB) can be more natural. So a model of dynamical supersymmetry breaking (DSB) which appears in one of these broad classes is naturally tied to a complementary mediation mechanism. Moreover, these mediation mechanisms have very important phenomenological consequences; for example, GMSB can provide an elegant explanation for small flavor-changing neutral currents. There is thus great interest in how this whole picture can be tied together in detail. For example, Aharony, Kachru and Silverstein exhibited models of "retrofitted" DSB which naturally appear in the context of branes at singularities [6]. 
On the other side, in [7] it was shown that intersecting brane models with non-trivial Fayet-Iliopoulos terms naturally generate SUSY-breaking in the open-string sector at a scale exponentially smaller than the FI-term scale. In this note, we consider an extension of the specific example of this class studied in [7], and examine the GMSB phenomenology (note, Cvetic and Weigand recently considered GMSB in a different IBM, which did not utilize nonvanishing FI-terms [8]; gauge-mediation in string models was also considered in [9]). In particular, we find that scalar vevs break gauge symmetries at a relatively high "natural" scale $\sqrt{\xi}$ set by the Fayet-Iliopoulos terms. But the SUSY-breaking $F$-terms are further suppressed by exponentially small couplings in the superpotential, $\langle F\rangle \sim \lambda \xi$. The gauge messengers then get masses $\sim \lambda \sqrt{\xi}$, yielding soft masses which are suppressed from the natural scale by $m_{\text {soft }} \sim \frac{g^{2}}{16 \pi^{2}} \lambda \sqrt{\xi}$.

In section 2 we will describe the specific intersecting brane model and the details of dynamical supersymmetry breaking. In section 3 we will study the phenomenology of gauge-mediation in this model, and we will conclude with a discussion of further issues in section 4 .

\section{Model}

For any string model to have realistic low-energy phenomenology, it must somehow generate the chiral matter content of the Standard Model. In intersecting brane models, the light chiral matter lies at the intersection of D-branes which fill spacetime and wrap cycles of the six compact extra dimensions which are inherent to string theory. In Type IIA string theory constructions, these are D6-branes which fill spacetime and wrap a 3-cycle of an orientifolded Calabi-Yau 3-fold.

Essentially, the gauge theory degrees of freedom arise from strings which begin and end on the same stack of D-branes, while chiral matter transforming in the bifundamental representation of two gauge groups arises from strings stretching between two brane stacks at their point of intersection. In particular, the number of chiral multiplets transforming in the bifundamental of groups $G_{a}$ and $G_{b}$ living on D-brane stacks $a$ and $b$ is counted by $I_{a b}$, the topological intersection number of the two brane stacks ${ }^{1}$.

\footnotetext{
${ }^{1}$ Chiral multiplets transforming in symmetric and anti-symmetric representations will also arise from strings stretching between a brane and its orientifold image, but we will not need to deal with these multiplets here.
} 
In [7], it was pointed out that intersecting brane models can naturally exhibit dynamical supersymmetry breaking. The key features here are that the Fayet-Iliopoulos terms are non-zero and the Yukawa couplings $\lambda_{i}$ are exponentially small (because they are generated by worldsheet instantons). As a result, the configuration space is constrained to live on $D$-flat directions where the fields have vevs of order the FI-terms. The $F$-terms are then generically non-zero, and yield an $F$-term potential $V_{F} \sim \lambda^{2} \xi^{2}$ which is exponentially small compared to the natural scale of the gauge theory set by the FI-terms.

The particular example described in [7] had three $U(1)$ gauge groups, and three chiral multiplets which were each charged under two of the three gauge groups. The three $U(1)$ FI-terms were taken to be non-zero, and the three multiplets were coupled by a superpotential term with an exponentially suppressed Yukawa coupling.

Here, we generalize the above model to include the fields which mediate supersymmetry breaking to a Standard Model sector via gauge interactions. The brane construction is depicted in Fig. 1, and it is assumed (for simplicity) that all brane stacks generate $U(1)$ gauge groups. The intersection numbers of the various brane stacks are given by: $I_{b a}=I_{a c}=I_{c b}=I_{a d}=I_{d b}=I_{a f}=$ $I_{g b}=I_{f e}=I_{e g}=1 ; I_{e a}=I_{b e}=2$ (all others are zero). The field content and associated charges are summarized in Table 1.

In this setup, brane stacks $a, b$ and $c$ are the dynamical SUSY-breaking sector described in [7]. The brane stack $d$ is a Standard Model brane stack. In an SU(5) GUT model, it could be a brane stack where $U(5)_{G U T}$ lives. Otherwise, it could be a stack where $S U(2)_{L}$ lives. The chiral multiplets $M_{1,2}$ are the gauge messengers; although they are non-vectorlike under hidden sector gauge groups, they are vectorlike under the Standard Model. The remaining branes are included to ensure that the superpotential couplings are sufficiently generic and that cubic anomalies are canceled (equivalently, that there are no RR-tadpoles) ${ }^{2}$.

Without loss of generality, we may assume $\left|\eta_{a}\right|^{2}=\left|\eta_{a}^{1}\right|^{2}+\left|\eta_{a}^{2}\right|^{2}$. Given the

\footnotetext{
${ }^{2}$ In general, more than one brane stack would be required to construct a visible sector. In this toy model the additional branes are not relevant to the SUSY-breaking phenomenology. If the additional SM branes intersect the branes of the DSB sector, then the superpotential will be more complicated. For the reasons described above and in 7 , we still expect supersymmetry to be dynamically broken, but obtaining reasonable phenomenology might be more complicated. We will assume no such intersections here.
} 


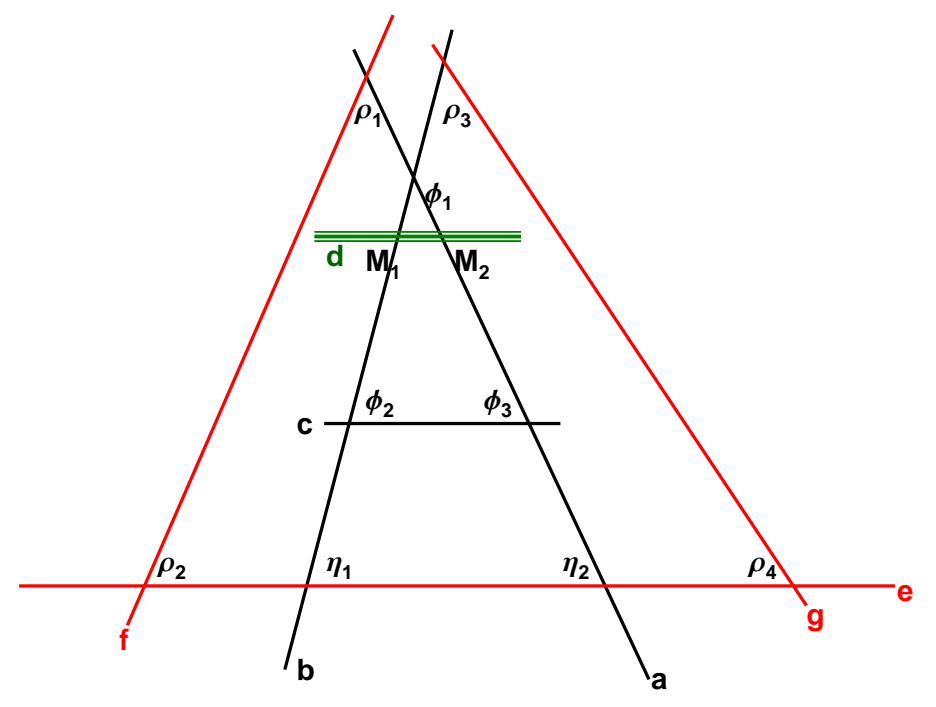

Figure 1: This figure depicts the various brane stacks of this model intersecting in the extra dimensions. The brane stack $d$ is a visible sector MSSM brane. The brane stacks $a, b$ and $c$ are responsible for the relevant dynamical supersymmetry breaking. the remaining stacks $e, f$ and $g$ are added to ensure that cubic anomalies are canceled. The multiplets shown in the figure arise from strings living at the indicated topological intersections.

charges of the various scalar fields, the general $D$-term potential is given by

$$
\begin{aligned}
V_{D}= & \frac{g_{a}^{2}}{2}\left(\left|\phi_{3}\right|^{2}-\left|\phi_{1}\right|^{2}+\left|M_{2}\right|^{2}-\left|\eta_{2}\right|^{2}+\left|\rho_{1}\right|^{2}-\xi_{a}\right)^{2} \\
& +\frac{g_{b}^{2}}{2}\left(\left|\phi_{1}\right|^{2}-\left|\phi_{2}\right|^{2}-\left|M_{1}\right|^{2}+\left|\eta_{1}\right|^{2}-\left|\rho_{3}\right|^{2}-\xi_{b}\right)^{2} \\
& +\frac{g_{c}^{2}}{2}\left(\left|\phi_{2}\right|^{2}-\left|\phi_{3}\right|^{2}-\xi_{c}\right)^{2}+\frac{g_{d}^{2}}{2}\left(\left|M_{1}\right|^{2}-\left|M_{2}\right|^{2}-\xi_{d}\right)^{2} \\
& +\frac{g_{e}^{2}}{2}\left(\left|\eta_{2}\right|^{2}-\left|\eta_{1}\right|^{2}+\left|\rho_{4}\right|^{2}-\left|\rho_{2}\right|^{2}-\xi_{e}\right)^{2} \\
& +\frac{g_{f}^{2}}{2}\left(\left|\rho_{2}\right|^{2}-\left|\rho_{1}\right|^{2}-\xi_{f}\right)^{2}+\frac{g_{g}^{2}}{2}\left(\left|\rho_{3}\right|^{2}-\left|\rho_{4}\right|^{2}-\xi_{g}\right)^{2}
\end{aligned}
$$


Table 1: Field Content

\begin{tabular}{cccccccc} 
& $U(1)_{a}$ & $U(1)_{b}$ & $U(1)_{c}$ & $U(1)_{d}$ & $U(1)_{e}$ & $U(1)_{f}$ & $U(1)_{g}$ \\
\hline$\phi_{1}$ & -1 & +1 & 0 & 0 & 0 & 0 & 0 \\
$\phi_{2}$ & 0 & -1 & +1 & 0 & 0 & 0 & 0 \\
$\phi_{3}$ & +1 & 0 & -1 & 0 & 0 & 0 & 0 \\
$M_{1}$ & 0 & -1 & 0 & +1 & 0 & 0 & 0 \\
$M_{2}$ & +1 & 0 & 0 & -1 & 0 & 0 & 0 \\
$\eta_{1}^{1,2}$ & 0 & +1 & 0 & 0 & -1 & 0 & 0 \\
$\eta_{2}^{1,2}$ & -1 & 0 & 0 & 0 & +1 & 0 & 0 \\
$\rho_{1}$ & +1 & 0 & 0 & 0 & 0 & -1 & 0 \\
$\rho_{2}$ & 0 & 0 & 0 & 0 & -1 & +1 & 0 \\
$\rho_{3}$ & 0 & -1 & 0 & 0 & 0 & 0 & +1 \\
$\rho_{4}$ & 0 & 0 & 0 & 0 & +1 & 0 & -1
\end{tabular}

The Fayet-Iliopoulos terms $\xi_{a, \ldots, g}$ can be non-zero, and control the mass of the lightest scalar excitations of the strings stretching between different brane stacks (in the Type IIA picture, they are set by the angles between the D6brane stacks and the orientifold planes [10]). The $\xi_{a \ldots g}$ need not be identical, but in the interests of simplicity of notation and of the model, we will assume the non-zero FI-terms are all at about the same approximate scale $\xi$. The FI-terms are naturally set by the string scale; however, if they are in fact at the string scale, the effective field theory is not in a controlled regime (since the excited string excitations will be at the same scale as light chiral modes). To ensure that the effective field theory approximation is valid, we will assume that the scale of the FI-terms $\xi$ is slightly suppressed from the string scale; the suppression need not be large. Indeed, it would be quite reasonable to set the $\xi$ at the GUT scale, if the string scale is somewhere between the GUT and Planck scales 3 .

For the superpotential, we take

$$
W=\lambda_{1} \phi_{1} M_{1} M_{2}+\lambda_{2} \phi_{1} \phi_{2} \phi_{3}+\lambda_{3}^{i} \eta_{1}^{i} \rho_{3} \rho_{4}+\lambda_{4}^{i} \eta_{2}^{i} \rho_{1} \rho_{2}+\lambda_{t} \xi_{t} \phi_{1}
$$

where $i=1,2$. We will assume for simplicity that $\lambda_{3}^{1}=\lambda_{3}^{2}, \lambda_{4}^{1}=\lambda_{4}^{2}$. The first four terms constitute the most general renormalizable superpotential

\footnotetext{
${ }^{3}$ In string theory, these FI-terms in fact depend on closed-string moduli. They may be treated as constants only if (as we assume) those moduli are stabilized at a scale higher than the gauge-theory scale [11].
} 
which is classically gauge-invariant. These Yukawa couplings are generated by Euclidean worldsheet instantons which stretch between the three brane stacks that the coupled fields begin and end on [12]. Importantly, the Yukawa couplings are exponentially suppressed by the area of the instanton, $\lambda \propto$ $e^{-A / l_{s}^{2}}$. In particular, in the limit where the extra dimensions are larger than the string scale (which is where most mechanisms for moduli stabilization are effective), these Yukawa couplings are expected to be exponentially small ${ }^{4}$.

In addition to these cubic terms, there may be terms in the superpotential which naively are not gauge-invariant. These terms can arise because the $U(1)$ symmetries suffer from mixed anomalies, due to the presence of matter transforming in non-vectorlike representations. In string theory, these mixed anomalies are fixed by the Green-Schwarz mechanism; a closed-string axion field shifts under the anomalous $U(1)$ and causes a classical transformation of the action which cancels the anomalous loop contribution. But if this axion field appears in the exponent of a superpotential coupling, then it generates a phase which can cancel the naively gauge-non-invariant phase arising from the open string scalars. This field is absorbed into the coupling in the low-energy limit, below the scale where closed-string moduli are stabilized. The last term in the superpotential is such a term, where $\xi_{t}$ is an energy scale which is chosen to be of the same order as the FI-terms (we may do this without loss of generality by rescaling the coupling; note that the hierarchy between the scale $\xi$ and the string scale is assumed to be relatively small). Because this coupling is generated by an E2-instanton, it is also non-perturbatively small [13, 8]. The essential point about the superpotential we have written is that, in terms of the natural scale $\xi$, all terms in the superpotential have dimensionless coefficients which are exponentially small (and in particular, much smaller than the gauge couplings $g_{a, \ldots, g}$ ). We will take all of the $\lambda$ 's to be real and positive.

For simplicity, we assume $\xi_{d}=0$. Otherwise, we would be forced to give a vev to the messengers and break SM or GUT symmetry at this stage. Although this might be desirable in certain GUT models, we will avoid this complication $5^{5}$. We have $\sum_{i=a, \ldots, g} \xi_{i}=0$, and we will assume that $\xi_{e, g}<0$ and

\footnotetext{
${ }^{4}$ Note that in any intersecting brane model, it is necessary for the top Yukawa coupling to be $\mathcal{O}(1)$. This can arise from a worldsheet instanton which is of string-scale size, which would require a modest tuning if the compact dimensions are only moderately large.

${ }^{5}$ In a realistic model, $U(1)_{Y}$ should be FI-free. This can arise if $U(1)_{Y}$ is a subgroup of a GUT group (like $S U(5)$ ), or if $U(1)_{Y}$ arises as the FI-free linear combination of various $U(1)$ 's living on various D-branes
} 
$\xi_{a, b, c}>0$ (this particular choice will yield interesting realistic phenomenology; there may be other choices which also work).

Because the superpotential couplings are all exponentially suppressed, the field configuration will sit close to a $D$-flat direction, but will be shifted away from the $D$-flat direction by corrections which are suppressed by $\mathcal{O}\left(\lambda_{i}^{2}\right)$. One solution to the $D$-term equations is given by

$$
\begin{aligned}
\left|\phi_{1}\right|^{2} & =\xi_{b}+\xi_{c} \\
\left|\phi_{2}\right|^{2} & =\xi_{c} \\
\left|\rho_{1}\right|^{2} & =\xi_{a}+\xi_{b}+\xi_{c} \\
\left|\rho_{2}\right|^{2} & =-\xi_{e}-\xi_{g} \\
\left|\rho_{4}\right|^{2} & =-\xi_{g} \\
\phi_{3}=\eta_{1}^{i}=\eta_{2}^{i}=M_{1}=M_{2}=\rho_{3} & =0
\end{aligned}
$$

There are $5 D$-flat directions away from this solution:

$$
\begin{aligned}
\delta\left|\phi_{1}^{2}\right|=\delta\left|\phi_{2}\right|^{2}=\delta\left|\phi_{3}\right|^{2} & =r_{1}, \\
\delta\left|\phi_{1}^{2}\right|=\delta\left|M_{1}\right|^{2}=\delta\left|M_{2}\right|^{2} & =r_{2}, \\
-\delta\left|\phi_{1}^{2}\right|=\delta\left|\eta_{1}\right|^{2}=\delta\left|\eta_{2}\right|^{2} & =r_{3}, \\
\delta\left|\rho_{1}^{2}\right|=\delta\left|\rho_{2}\right|^{2}=\delta\left|\eta_{2}\right|^{2} & =r_{4}, \\
\delta\left|\rho_{3}^{2}\right|=\delta\left|\rho_{4}\right|^{2}=\delta\left|\eta_{1}\right|^{2} & =r_{5},
\end{aligned}
$$

where we are constrained to $r_{1, \ldots, 5} \geq 0$. The $\lambda_{3}$ terms in $V_{F}$ force the field configuration to $r_{5}=0$. Similarly, the $\lambda_{4}$ terms force us to $r_{4}=0$. However, $r_{1,2,3}$ are not yet constrained. Plugging in the expansion above and keeping terms linear in $r_{1,2,3}$ gives

$$
\begin{aligned}
V_{F}= & \left|\lambda_{1} M_{1} M_{2}+\lambda_{2} \phi_{2} \phi_{3}+\lambda_{t} \xi_{t}\right|^{2}+\lambda_{2}^{2}\left|\phi_{1}\right|^{2}\left|\phi_{2}\right|^{2}+\lambda_{2}^{2}\left|\phi_{1}\right|^{2}\left|\phi_{3}\right|^{2} \\
& +\lambda_{1}^{2}\left|\phi_{1}\right|^{2}\left|M_{1}\right|^{2}+\lambda_{1}^{2}\left|\phi_{1}\right|^{2}\left|M_{2}\right|^{2}+\lambda_{3}^{2}\left|\eta_{1}\right|^{2}\left|\rho_{4}\right|^{2}+\lambda_{3}^{2}\left|\eta_{1}\right|^{2}\left|\rho_{3}\right|^{2} \\
& +\lambda_{3}^{2}\left|\rho_{3}\right|^{2}\left|\rho_{4}\right|^{2}+\lambda_{4}^{2}\left|\eta_{2}\right|^{2}\left|\rho_{1}\right|^{2}+\lambda_{4}^{2}\left|\eta_{2}\right|^{2}\left|\rho_{2}\right|^{2}+\lambda_{4}^{2}\left|\rho_{1}\right|^{2}\left|\rho_{2}\right|^{2} \\
\sim & \lambda_{4}^{2}\left|\left(\xi_{a}+\xi_{b}+\xi_{c}\right)\left(\xi_{e}+\xi_{g}\right)\right|+\left|\lambda_{t} \xi_{t}\right|^{2}+\lambda_{2}^{2}\left|\xi_{c}\left(\xi_{b}+\xi_{c}\right)\right| \\
& +\left[\lambda_{2}^{2}\left(2 \xi_{b}+4 \xi_{c}\right) r_{1}-2 \lambda_{2} \lambda_{t}\left|\xi_{t}\right| \sqrt{\xi_{c}} \sqrt{r_{1}}\right] \\
& +\left[\lambda_{2}^{2} \xi_{c}+2 \lambda_{1}^{2}\left(\xi_{b}+\xi_{c}\right)-2 \lambda_{1} \lambda_{t}\left|\xi_{t}\right|\right] r_{2} \\
& +\left[\lambda_{3}^{2}\left|\xi_{g}\right|+\lambda_{4}^{2}\left(\xi_{a}+\xi_{b}+\xi_{c}-\xi_{e}-\xi_{g}\right)-\lambda_{2}^{2} \xi_{c}\right] r_{3}
\end{aligned}
$$

The potential will force $r_{2,3}=0$ if the following two constraints are satisfied: 
- $\lambda_{2}^{2} \xi_{c}+2 \lambda_{1}^{2}\left(\xi_{b}+\xi_{c}\right)>2 \lambda_{1} \lambda_{t}\left|\xi_{t}\right|$

- $\lambda_{3}^{2}\left|\xi_{g}\right|+\lambda_{4}^{2}\left(\xi_{a}+\xi_{b}+\xi_{c}-\xi_{e}-\xi_{g}\right)>\lambda_{2}^{2} \xi_{c}$

Miminizing the potential with respect to $\sqrt{r_{1}}$ gives

$$
\sqrt{r_{1}}=\frac{\lambda_{t}}{\lambda_{2}} \frac{\xi_{t} \sqrt{\xi_{c}}}{2\left(\xi_{b}+2 \xi_{c}\right)} .
$$

If $\sqrt{r_{1}}$ is small, then the linear approximation used above is valid. Clearly, one can satisfy all three constraints (for example, if all $\xi$ are of the same order and $\left.\lambda_{t} \ll \lambda_{2} \ll \lambda_{3}\right)$. Supersymmetry is then broken, and the non-zero F-terms are

$$
\begin{aligned}
F_{\eta_{2}^{i}} & =\lambda_{4}^{i} \sqrt{\xi_{a}+\xi_{b}+\xi_{c}} \sqrt{\left|\xi_{e}+\xi_{g}\right|} \\
F_{\phi_{1}} & =\lambda_{t} \xi_{t}\left(1-\frac{\xi_{c}}{2\left(\xi_{b}+2 \xi_{c}\right)}\right) \\
F_{\phi_{2}} & =\lambda_{t} \frac{\xi_{t} \sqrt{\xi_{c}\left(\xi_{b}+\xi_{c}\right)}}{2\left(\xi_{b}+2 \xi_{c}\right)} \\
F_{\phi_{3}} & =\lambda_{2} \sqrt{\xi_{c}} \sqrt{\xi_{b}+\xi_{c}}+\mathcal{O}\left(\lambda_{t} \xi\right) .
\end{aligned}
$$

Non-zero $D$-terms arise because the field configuration is shifted away from a $D$-flat direction by corrections due to the $F$-term potential. But the $D$-terms scale as

$$
V_{D} \sim \mathcal{O}\left(\frac{\lambda^{4}}{g^{2}} \xi^{2}\right)
$$

and are much smaller than the $F$-terms in the limit where the Yukawa couplings are exponentially suppressed [7].

\section{Phenomenology}

In the model we have described, the multiplets $M_{1,2}$ naturally act as gauge messengers, which are charged under both the MSSM sector and the SUSYbreaking sector. The mass-splitting between the bosonic and fermionic components of the messenger multiplets is generated by the superpotential term

$$
W=\lambda_{1} \phi_{1} M_{1} M_{2}+\ldots
$$


As such, the $F$-term relevant for GMSB phenomenology in this model is $F_{\phi_{1}}$. We can compute the soft scalar masses and find

$$
m_{\text {scalar }} \sim \frac{g^{2}}{16 \pi^{2}} \frac{\lambda_{1} F_{\phi_{1}}}{\lambda_{1}\left\langle\phi_{1}\right\rangle} \sim \frac{g^{2} \lambda_{t}}{16 \pi^{2}} \frac{\xi_{t}}{\sqrt{\xi_{b}+\xi_{c}}}\left(1-\frac{\xi_{c}}{2\left(\xi_{b}+2 \xi_{c}\right)}\right)
$$

So if all $\xi$ are of the same natural scale, then the soft scalars are at $m^{2} \sim g^{4} \lambda_{t}^{2} \xi$, and are exponentially suppressed with respect to the scale $\xi$. Furthermore, we have $W=\lambda_{t} \xi_{t} \sqrt{\xi_{b}+\xi_{c}}$, so $R$-symmetry is broken and the gauginos get a mass at the same scale as the scalars (for this purpose, it was important that in our solution $\left.\left\langle\phi_{1}\right\rangle,\left\langle F_{\phi_{1}}\right\rangle \neq 0\right)$. The gravitino mass is given by $m_{3 / 2} \sim \frac{F}{M_{p l}}$, but in the limit we took earlier, the dominant $F$-term is $F_{\phi_{3}} \sim \lambda_{2} \xi$. We would thus find

$$
\begin{aligned}
& m_{3 / 2} \sim \frac{F_{\phi_{3}}}{M_{P l}} \sim \lambda_{2} \frac{\xi}{M_{P l}} . \\
& \frac{m_{3 / 2}^{2}}{m_{0}^{2}} \sim \frac{256 \pi^{4} \lambda_{2}^{2}}{g^{4} \lambda_{t}^{2}} \frac{\xi}{M_{P l}^{2}} .
\end{aligned}
$$

To get a feel for the phenomenology of a somewhat natural example, let us assume that the scale of the FI-terms is $\xi \sim\left(10^{15} \mathrm{GeV}\right)^{2}$, while the squark and slepton masses are $\sim \mathrm{TeV}$. Then from the above formula we would find that $\lambda_{t} \sim 10^{-10}$. This is not unreasonable if the extra dimensions are (as is typical) stabilized at scales which are somewhat larger than the string scale [14], as the coupling is generated by an E2-instanton [13] which is suppressed by $\exp \left[-\frac{2 \pi}{l_{s}^{3} s_{s}} V o l_{E 2}\right]$. Larger Yukawa couplings could be accommodated by having a smaller scale $\xi$. Note that the messenger mass scale is $m_{\text {mess. }} \sim \lambda_{1} \xi$; since we have not yet constrained the $\lambda_{1}$ coupling, messenger masses from $\mathrm{TeV}$ to just below GUT scale are possible.

Assuming the point in parameter-space we described above, we then find

$$
m_{3 / 2} \sim \lambda_{2} \times 10^{11} \mathrm{GeV} .
$$

Given our assumption $\lambda_{2}>\lambda_{t}$, we would find in this model a relatively heavy gravitino with $m_{3 / 2}>10 \mathrm{GeV}$. In particular, the gravitinos here are typically heavier than those found in [8]. This seems quite reasonable; since the natural scale of the theory is well above the intermediate scale, even with suppressions of the $F$-terms from Yukawa couplings, one would expect gravitational interactions to be relatively large. It might be even more natural 
to have a slightly higher scale for $\xi$, say at $10^{16} \mathrm{GeV}$. However, in that case the gravity-mediated contributions become significant enough that they can potentially violate FCNC constraints. Since, absent an model-dependent explanation, there is no reason for gravity contributions to respect flavor, it is safest to allow a small hierarchy between $\xi$ and the GUT scale.

In GMSB, gravitinos have a very interesting effect on cosmology. If Rsymmetry is preserved, gravitinos are stable and one must be sure that they do not overclose the universe. For gravitinos in the mass-range discussed here, the dominant production mechanism is through sparticle scattering [16], and one finds that the gravitino density would be phemenologically acceptable for reheat temperatures $T_{R}<10^{8-9} \mathrm{GeV}$ (or, alternatively, if there were some mechanism of late entropy production).

It is worth noting also that in this model we have introduced only one pair of messenger multiplets. Although vectorlike matter transforming in complete GUT group representations will not prevent gauge coupling unification, they will change the scale at which it occurs. If enough matter multiplets are added, the couplings may become strong before unification, making the gauge-coupling unification unreliable. One of the advantages of SUSY-breaking sectors with Abelian dynamics (such as the one described here) is that they typically arise with small numbers of messengers, avoiding this problem.

\section{Discussion}

We have exhibited a toy example of an intersecting brane model in which supersymmetry is dynamically broken in an open string hidden sector, and transmitted to the visible SM sector via gauge interactions. The natural scale of the theory is set by the scale of the Fayet-Iliopoulos terms, but the SUSY-breaking $F$-terms are at an exponentially smaller scale. The soft masses in the visible sector receive additional suppression from a loop factor, and with reasonable choices of parameters the gravitino is moderately lighter than scalars. This hierarchy of scales is summarized in Table 2.

It is interesting to note that this work illustrates a point made in [7]; if FI-terms are turned on, one generically expects F-term SUSY-breaking at exponentially small scales in intersecting brane models. Although the model presented here extends the model in [7] by adding more fields and couplings, the DSB result is robust. Moreover, it was quite easy to obtain realistic 
Table 2: Scales

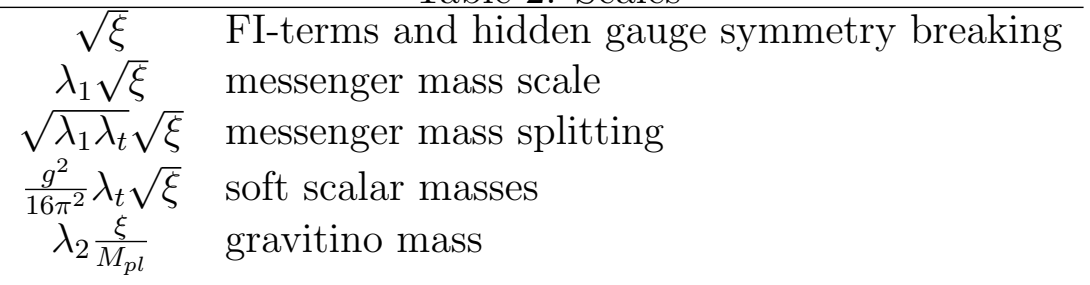

GMSB phenomenology.

There are a number of open questions worth studying. From a formal standpoint, perhaps the most interesting questions are related to modulistabilization. In the framework described above, we have essentially treated the FI-terms as constants. But in string theory, the FI-terms depend on closed string moduli (for example, in the Type IIA picture, the FI-terms are set by the relative angles between the D6-branes, and thus depend on the complex structure moduli). For these parameters to be approximately constant, they must be stabilized at a scale which is much larger than the scale of the gauge theory. It has been argued that this can be done in a controlled manner [15], but we have not utilized any particular moduli stabilization scheme. It would be very interesting to actually construct a phenomenologically viable model of gauge-mediated dynamical supersymmetry breaking where these issues of moduli-stabilization are concretely addressed.

In is interesting to note that the relative hierarchies at phenomenological scales (say, between $m_{3 / 2}$ and $m_{0}$ ) are controlled by different Yukawa couplings. As a result, large mass hierarchies are obtainable from relatively small hierarchies in areas in the extra dimensions. This implies that detailed phenomenology in these types of models can depend crucially on how moduli and brane positions are stabilized in the extra dimensions. It would be very interesting to understand how these issues can be addressed concretely in orientifolded Calabi-Yau 3-folds compactifications.

\section{Acknowledgments}

This work was supported by NSF grants PHY-0239817, PHY-0314712 and PHY-0653656. We are grateful to J. Feng, S. Franco, A. Rajaraman, B. Thomas and J. Wells for useful discussions, and to S. Kachru and E. Silverstein for collaboration at an early stage of this project. 


\section{References}

[1] For reviews, see I. Affleck, M. Dine and N. Seiberg, Nucl. Phys. B 256, 557 (1985); E. Poppitz and S. P. Trivedi, Ann. Rev. Nucl. Part. Sci. 48, 307 (1998) arXiv:hep-th/9803107]; Y. Shadmi and Y. Shirman, Rev. Mod. Phys. 72, 25 (2000) [arXiv:hep-th/9907225]; K. Intriligator and N. Seiberg, arXiv:hep-ph/0702069. See also M. Cvetic, P. Langacker and J. Wang, Phys. Rev. D 68, 046002 (2003) arXiv:hep-th/0303208.

[2] K. Intriligator, N. Seiberg and D. Shih, JHEP 0604, 021 (2006) arXiv:hep-th/0602239.

[3] For example, see H. Ooguri and Y. Ookouchi, Phys. Lett. B 641, 323 (2006) arXiv:hep-th/0607183; R. Argurio, M. Bertolini, S. Franco and S. Kachru, JHEP 0706, 017 (2007) |arXiv:hep-th/0703236]; K. Intriligator, N. Seiberg and D. Shih, JHEP 0707, 017 (2007) arXiv:hepth/0703281; M. Aganagic, C. Beem and S. Kachru, Nucl. Phys. B 796, 1 (2008) arXiv:0709.4277 [hep-th]]; J. Marsano, K. Papadodimas and M. Shigemori, arXiv:0801.2154 [hep-th]; C. Beasley, J. J. Heckman and C. Vafa, arXiv:0806.0102 [hep-th]; K. R. Dienes and B. Thomas, arXiv:0806.3364 [hep-th]; J. J. Heckman, J. Marsano, N. Saulina, S. Schafer-Nameki and C. Vafa, arXiv:0808.1286 [hep-th].

[4] R. Kitano, H. Ooguri and Y. Ookouchi, Phys. Rev. D 75, 045022 (2007) [arXiv:hep-ph/0612139]; H. Murayama and Y. Nomura, Phys. Rev. Lett. 98, 151803 (2007) |arXiv:hep-ph/0612186]; H. Murayama and Y. Nomura, Phys. Rev. D 75, 095011 (2007) |arXiv:hep-ph/0701231|; T. Kawano, H. Ooguri and Y. Ookouchi, Phys. Lett. B 652, 40 (2007) arXiv:0704.1085 [hep-th]]; N. Haba and N. Maru, Phys. Rev. D 76, 115019 (2007) [arXiv:0709.2945 [hep-ph]]; M. Buican and S. Franco, arXiv:0806.1964 [hep-th].

[5] M. Dine, J. L. Feng and E. Silverstein, Phys. Rev. D 74, 095012 (2006) arXiv:hep-th/0608159].

[6] O. Aharony, S. Kachru and E. Silverstein, Phys. Rev. D 76, 126009 (2007) arXiv:0708.0493 [hep-th]].

[7] J. Kumar, Phys. Rev. D 77, 046010 (2008) arXiv:0708.4116 [hep-th]]. 
[8] M. Cvetic and T. Weigand, arXiv:0807.3953 [hep-th].

[9] E. Floratos and C. Kokorelis, arXiv:hep-th/0607217.

[10] M. Berkooz, M. R. Douglas and R. G. Leigh, Nucl. Phys. B 480, 265 (1996) arXiv:hep-th/9606139; N. Ohta and P. K. Townsend, Phys. Lett. B 418, 77 (1998) |arXiv:hep-th/9710129].

[11] K. Intriligator and N. Seiberg, JHEP 0602, 031 (2006) arXiv:hepth/0512347.

[12] G. Aldazabal, S. Franco, L. E. Ibanez, R. Rabadan and A. M. Uranga, JHEP 0102, 047 (2001) arXiv:hep-ph/0011132]; S. Kachru, S. H. Katz, A. E. Lawrence and J. McGreevy, Phys. Rev. D 62, 026001 (2000) arXiv:hep-th/9912151]; D. Cremades, L. E. Ibanez and F. Marchesano, JHEP 0307, 038 (2003) arXiv:hep-th/0302105]; M. Cvetic and I. Papadimitriou, Phys. Rev. D 68, 046001 (2003) [Erratum-ibid. D 70, 029903 (2004)] [arXiv:hep-th/0303083.

[13] O. J. Ganor, Nucl. Phys. B 499, 55 (1997) arXiv:hep-th/9612077]; R. Blumenhagen, M. Cvetic and T. Weigand, Nucl. Phys. B 771, 113 (2007) arXiv:hep-th/0609191]; L. E. Ibanez and A. M. Uranga, JHEP 0703, 052 (2007) (arXiv:hep-th/0609213|; B. Florea, S. Kachru, J. McGreevy and N. Saulina, JHEP 0705, 024 (2007) arXiv:hepth/0610003; M. Cvetic, R. Richter and T. Weigand, JHEP 0807, 012 (2008) [arXiv:0803.2513 [hep-th]].

[14] For reviews, see E. Silverstein, arXiv:hep-th/0405068; M. Grana, Phys. Rept. 423, 91 (2006) arXiv:hep-th/0509003); M. R. Douglas and S. Kachru, Rev. Mod. Phys. 79, 733 (2007) arXiv:hep-th/0610102; R. Blumenhagen, B. Kors, D. Lust and S. Stieberger, Phys. Rept. 445, 1 (2007) arXiv:hep-th/0610327]; F. Denef, M. R. Douglas and S. Kachru, Ann. Rev. Nucl. Part. Sci. 57, 119 (2007) arXiv:hep-th/0701050|.

[15] P. Binetruy, G. Dvali, R. Kallosh and A. Van Proeyen, Class. Quant. Grav. 21, 3137 (2004) arXiv:hep-th/0402046]; D. E. Diaconescu, R. Donagi and B. Florea, Nucl. Phys. B 774, 102 (2007) arXiv:hepth/0701104; K. Sinha, arXiv:0709.2932 [hep-th].

[16] G. F. Giudice and R. Rattazzi, Phys. Rept. 322, 419 (1999) arXiv:hepph/9801271]; J. L. Feng, Annals Phys. 315, 2 (2005). 